\title{
Distinct Physiological Mechanisms Induce Latitudinal and Sexual Differences in the Photoperiodic Induction of Diapause in a Fly
}

\section{Koki Yamaguchi, Shin G. Goto}

\begin{tabular}{|c|l|}
\hline Citation & Journal of Biological Rhythms, 34(3); 293-306 \\
\hline Issue Date & 2019-06-01 \\
\hline Type & Journal Article \\
\hline Textversion & author \\
\hline Rights & $\begin{array}{l}\text { The following article has been accepted by Journal of Biological Rhythms. After it is } \\
\text { published, it will be found at https://doi.org/10.1177/0748730419841931. This article } \\
\text { may be downloaded for personal use only. Any other use requires prior permission of } \\
\text { SAGE Publications. }\end{array}$ \\
\hline DOI & 10.1177/0748730419841931 \\
\hline
\end{tabular}

Self-Archiving by Author(s)

Placed on: Osaka City University 
Distinct physiological mechanisms induce latitudinal and sexual differences in the photoperiodic induction of

\author{
diapause in a fly
}

Koki Yamaguchi and Shin G. Goto*

Graduate School of Science, Osaka City University, Japan

*To whom all correspondence should be addressed: Shin G. Goto, Graduate School of Science, Osaka City University, 3-3-138 Sugimoto, Sumiyoshi-ku, Osaka 558-8585,

Japan; e-mail: shingoto@sci.osaka-cu.ac.jp; Tel.: +81-6-6605-2573; Fax.: +81-6-6605-2522

Running title: Physiological mechanisms underlying latitudinal and sexual differences in insect photoperiodism 
Abstract Many temperate insects enter diapause (dormancy) for overwintering in response to short days (long nights). A latitudinal cline in the critical day lengths for the photoperiodic induction of diapause has been reported in a variety of insect species. However, the physiological mechanisms underlying this cline have remained elusive. We approached this issue in the flesh fly Sarcophaga similis, in which the photoperiodic time measurement system meets the 'external coincidence model'. In this model, measuring day lengths depends on whether the photoinducible phase $\left(\varphi_{\mathrm{i}}\right)$, determined by a circadian clock, is exposed to light or not. First, we detected a clear latitudinal cline in the critical day lengths of flies collected from four localities at different latitudes. The phase positions of the $\varphi_{\mathrm{i}}$, which can be verified by night interruption photoperiods, also showed a clear latitudinal cline. This result supports the hypothesis that the latitudinal cline in the critical day lengths is produced by the difference in the phase positions of the $\varphi_{i}$ among different strains. A sexual difference in the critical day lengths for photoperiodic induction has also been detected in various species. In this study, a sexual difference in the critical day lengths was observed in the southern strains, but 
there was no sexual difference in the phase positions of the $\varphi_{\text {i. }}$ This result indicates that both sexes measure photoperiods in the same manner. Males are less sensitive than females to the light pulse given at the $\varphi_{i}$, suggesting a quantitative difference in the photoperiodic time measurement and counter systems. This study clearly reveals that distinct mechanisms induce latitudinal and sexual differences in the critical day lengths for the photoperiodic induction of diapause in a fly.

Keywords latitudinal cline, sexual difference, critical day length, photoperiodism, flesh fly, diapause, external coincidence model 
The tilt of the Earth's axis, from the plane of its orbit around the sun, causing seasons with dramatic environmental contrasts. Some seasons are suitable for development and reproduction, but others are not. Thus, most insects inhabiting the temperate zones enter diapause, a physiological state in which development or reproduction is suppressed or arrested, in response to short day lengths, to overcome adverse conditions. This ability for organisms to respond to the photoperiod is called photoperiodism (Nelson et al., 2010; Goto and Numata, 2014). For example, the flesh fly Sarcophaga similis, the focal species in this study, shows a clear photoperiodic response. They develop without interruption under long-day conditions, whereas they enter diapause at the pupal stage in response to short-day conditions (Tanaka et al., 2008), as reported in other sarcophagid species (Denlinger, 1971; Kurahashi and Ohtaki, 1979; Saunders, 1976; Vinogradova, 1976). Although it is well known that many insect species measure the night length, not the day length (Saunders, 2013), the term 'day length' is widely used in literature and thus we also followed this terminology in this paper.

There is little intraspecific variation in the stages at which diapause occurs, whereas there is great intraspecific variation in the timing of diapause entry. The timing is determined by the critical day length, which is the length of the light fraction, of the light-dark cycle, that separates a strong long-day response from a strong short- 
day response in the photoperiodic response curve (Fig. 1A). One of the intraspecific variations in the critical day length is found among geographic strains (Danilevskii 1965; Danks, 1987; Saunders 2002). For example, in an exhaustive survey, 41 geographical strains of Drosophila littoralis were collected from localities ranging from the Black Sea coast $\left(41.6^{\circ} \mathrm{N}\right)$ to northern Finland $\left(69.0^{\circ} \mathrm{N}\right)$, revealing geographic variations in the critical day length (Lankinen, 1986). In this case, the critical day lengths ranged from 11.6 to $20.3 \mathrm{~h}$ (from south to north), which were highly correlated with latitude, i.e. a clear latitudinal cline. Such a latitudinal cline, with the longer critical day lengths in the strains originating from higher latitudes, is observed in several insect species (Bradshaw and Lounibos 1977; Kimura et al., 1993; Lankinen et al., 2013; Lehmann et al., 2015; Paolucci et al., 2013; Shimizu and Kawasaki, 2001; Wang et al., 2012). In addition, the rapid evolution of changes in critical day lengths for several invasive species has been measured or inferred in the field, after colonisation (Bean et al., 2012; Gomi et al., 2007; Tanaka and Murata, 2016; Tanaka et al., 2015; Urbanski et al., 2012). The significance of the latitudinal cline in the critical day length can be explained in terms of ecological aspects. Days are longer at higher latitudes between the spring and autumn equinoxes, but the climate is colder, and summer ends earlier than in areas at lower latitudes. Therefore, insects at higher latitudes likely enter 
diapause earlier by responding to the longer photoperiod (Saunders, 2002; Tauber et al., 1986).

Diapause incidence and the critical day length that triggers diapause can also differ intraspecifically between males and females (Danks, 1987). Males are more likely to enter diapause than are females; i.e., males have a longer critical day length than females (Butler et al., 1985; Denlinger, 1972; Shimizu and Fujisaki, 2002; Wiklund et al., 1992). Despite obvious ecological advantages, diapause itself is a metabolically expensive life history strategy. The costs of diapause are commonly reflected in a lower post-diapause survival and reduced fecundity (Bradshaw et al., 1998; Ellers and van Alphen, 2002; Matsuo, 2006; Sadakiyo and Ishihara 2012ab). In the flesh fly Sarcophaga crassipalpis, Denlinger (1981) found that the risks associated with overwintering are greater in females than in males. Females that had been in diapause show lower reproductive success, whereas males that had been in diapause do not suffer severe reproductive consequences from entering diapause. This explains the propensity for females to escape from diapause induction in autumn, as delaying the onset of diapause while temperatures are still favourable allows further reproduction before the winter sets in. Thus, for females, the timing of diapause inception becomes a trade-off between high fertility and environmental security. Wiklund et al. (1992) explained the significance of this sexual difference, from the view of evolutionarily stable strategies. 
In temperate areas where many insects have multiple generations, males should enter diapause at an earlier date than females should, because late-emerging males are penalised in terms of fewer mating opportunities.

We understand the ecological significance of the latitudinal and sexual differences in the critical day length for the photoperiodic induction of diapause. However, little is known about the physiological mechanisms underlying the differences (Hut et al., 2013). Photoperiodism is operated by several physiological modules including (i) photoreception, which extracts light information from environmental conditions; (ii) the photoperiodic time measurement system, which measures day or night length; (iii) the counter system, which accumulates photoperiodic information; and (iv) the endocrine system, which regulates synthesis and/or the secretion of neurohormones and/or hormones to reflect the photoperiodic information into a physiological state (Goto and Numata, 2014). Latitudinal and sexual differences in the critical day length can be derived from some modulations of the photoperiodic time measurement system and/or its downstream cascades. To determine the causal mechanisms regulating the intraspecific variation in critical day length, we used a flesh fly species, which shows a clear photoperiodic response and has been used for understanding insect photoperiodism. 
Sarcophaga similis is widely distributed in Europe and Asia. The photoperiodic time measurement for diapause induction in Sarcophaga species meets the 'external coincidence model' (Gnagey and Denlinger, 1984; Goto and Numata, 2009; Lewis and Saunders, 1987; Saunders, 1976, 1978a,b, 1979; Saunders and Lewis, 1987ab). This model hypothesises the involvement of an endogenous circadian oscillator in photoperiodic time measurement and the existence of a photoinducible phase $\left(\varphi_{\mathrm{i}}\right)$, which is located in the late phase of the subjective night under the control of a circadian clock (Pittendrigh and Minis, 1964; Fig. 1B). The $\varphi_{\mathrm{i}}$ is important for the determination of the developmental program. Short-day responses are produced when $\varphi_{\mathrm{i}}$ is in darkness, whereas long-day responses are produced when $\varphi_{\mathrm{i}}$ is exposed to light. During the summer, $\varphi_{i}$ is pushed out to the photophase because of the phase delay of the clock and thus $\varphi_{i}$ falls in the light period, representing a longer day to insects and accordingly eliciting a long-day response. Under autumnal short-day conditions, $\varphi_{i}$ falls in the dark period, which is interpreted as a short day and induces a short-day response. When a light pulse systematically scans the scotophase under short-day conditions, 2 light-sensitive phases can be observed (Goto and Numata, 2009; Gnagey and Denlinger, 1984; Tagaya et al., 2010; Fig. 1B). The phase located during the early scotophase is derived from the phase delay of the circadian clock, whereas the phase located during the late scotophase is the $\varphi_{\mathrm{i}}$ (Fig. 1B; Saunders, 2002). 
Insects, including flesh fly species, evaluate photoperiods quantitatively. This can be explained by the 'quantitative production of a diapause-inducing substance' (Tagaya et al., 2010). In this concept, the $\varphi_{\mathrm{i}}$ is not a short period of time but instead is much longer. Insects synthesise the hypothetical 'diapause-inducing substance' based on how long the $\varphi_{\mathrm{i}}$ is exposed to light. The synthetic rate of the substance is higher during shorter days but lower during longer days (or, the degradation rate of the substance is higher during longer days) (Fig. 1C). The substance accumulates in the counter system. When the level of the accumulated substance exceeds a certain 'internal threshold', diapause induction is determined, whereas nondiapause development is determined when the accumulation is lower than the threshold (Gibbs, 1975; Saunders, 1966; Fig. 1D). Although this concept sufficiently explains the quantitative discrimination of photoperiods, no such product has yet been verified. Latitudinal and sexual differences in critical day length (Fig. 2A) may be explained by the qualitative differences in photoperiodic time measurements, i.e. the phase-positional difference of the $\varphi_{i}$ (Hypothesis I, Fig. 2B; Saunders 2013). If the $\varphi_{i}$ of a group is located at an earlier point in the scotophase than that of another group, the critical day length of the former will be longer than that of the latter. This hypothesis can be explained by 2 models; the same phase angle of the $\varphi_{\mathrm{i}}$ in circadian clocks with different periods (Model A shown in Fig. 2B1-3; Hut and Beersma, 2011) and the 
different phase angles of the $\varphi_{\mathrm{i}}$ in circadian clocks with the same periods (Model B shown in Fig. 2B4-6). In the model A, the $\varphi_{i}$ resides in the same position (the same phase angle) in the circadian clocks, but the period of the clock is longer in a group with a short critical day length than in a group with a long critical day length. In the model B, the periods of the clocks are identical between the groups, but the $\varphi_{i}$ resides in the different positions (the different phase angle) in the circadian clocks. Such positional differences of the $\varphi_{\mathrm{i}}$ can be assessed by the systematic scanning of the scotophase using a short light pulse; i.e. the night interruption photoperiod (Fig. 2B7). Alternatively, the difference in the critical day length might be explained by the quantitative difference in the photoperiodic time measurement and counter systems (Hypothesis II, Fig. 2C; Tagaya et al., 2010). In this hypothesis, there is no positional difference of the $\varphi_{\mathrm{i}}$, but intraspecific variation in the synthetic rate of the diapauseinducing substance is assumed. If a group with a longer critical day length synthesises the substance at a higher rate than another group with a shorter critical day length, the substance is accumulated at a higher rate in the counter system of the former than in that of the latter. Thus, even under the same photoperiodic conditions, the short-day response is easier to be elicited in the former than in the latter. The basic concept of Hypothesis II is whether the accumulated amount of the substance exceeds the 
threshold or not, and thus, the hypothesis includes the same synthetic rates of the substance with different internal thresholds between the groups.

Tagaya et al. (2010) reported that a mid-latitude strain of S. similis shows sexual differences in the critical day length for diapause induction, which is that the critical day length of the males is longer than that of the females. The phase positions of the $\varphi_{\mathrm{i}}$ are identical between the sexes, whereas males are less sensitive to the light pulse given at the $\varphi_{\text {i. }}$ These results align with Hypothesis II (Fig. 2C). To expand our knowledge of the physiological mechanisms underlying photoperiodism, we focus on the latitudinal cline of the critical day lengths for diapause induction in S. similis. In this study, we collected 4 populations from different latitudes in Japan and compared their photoperiodic response curves to clarify their critical day lengths. A light pulse was emitted during the scotophase to find the phase position of the $\varphi_{i}$. We further investigated the sexual differences in the phase position of the $\varphi_{i}$ in these four geographic strains, to assess the similarity between latitudinal and sexual variations in the critical day lengths for the photoperiodic induction of diapause. 


\section{MATERIALS AND METHODS}

\section{Insects}

Adult flies of S. similis Meade were collected in Otaru City, Hokkaido Prefecture (OTR; $\left.43.20^{\circ} \mathrm{N}, 141.99^{\circ} \mathrm{E}\right)$; Utsunomiya City, Tochigi Prefecture (UTM; $\left.36.57^{\circ} \mathrm{N}, 139.90^{\circ} \mathrm{E}\right)$; Osaka City, Osaka Prefecture (OSK; $\left.34.59^{\circ} \mathrm{N}, 135.51^{\circ} \mathrm{E}\right)$; and

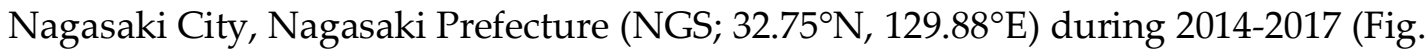
3A). Stock cultures of these strains were maintained under diapause-averting long-day conditions, $16 \mathrm{~h}$ of light and $8 \mathrm{~h}$ of darkness (LD 16:8 h) at $20 \pm 1{ }^{\circ} \mathrm{C}$ or $25 \pm 1{ }^{\circ} \mathrm{C}$.

For the experiments, adult flies were maintained in groups of less than 50 in a plastic container $(15 \mathrm{~cm}$ in diameter, $9 \mathrm{~cm}$ in depth) covered with nylon netting. Water, two pieces of block sugar, and a piece of beef or chicken liver were provisioned at $20 \pm$ $1{ }^{\circ} \mathrm{C}$ under the experimental photoperiodic conditions. Fifteen days after the first provision of the liver, a new piece of liver was provided as a larviposition site. The next day, larvae on the liver were collected and transferred to a chunk of liver on an aluminium dish. The dish was placed on dry wood chips (30-50 $\mathrm{mm}$ in depth, pupariation/pupation substrate) in a plastic container. Most larvae ceased feeding 5 days after being transferred, left their food, and get into the wood chips for pupariation. A few days later, they pupariate and pupate. 
White fluorescent lights (FLR15W, FLR20SW/M, FLR30SW, or FL40SW;

Panasonic, Kadoma, Osaka, Japan) were used as a white light source at an intensity ranging from 0.3 to $1.3 \mathrm{~W} \cdot \mathrm{m}^{-2}$ on the surface of the wood chips in the photophase of complete photoperiods, the main photophase, and the light pulse of night interruption photoperiods. However, the larvae were located within their food (liver) during the feeding stage and the wandering larvae were concealed in dry wood chips at a depth of 30-50 mm or in wet wood chips to a depth of $10 \mathrm{~mm}$ (see below). Therefore, the light intensity that the larvae received was dependent on their position.

\section{Photoperiodic response curves}

Insects were reared continuously under the experimental photoperiodic conditions, from their embryonic stage, to assess their diapause status. We adopted photoperiods yielding 0-100\% diapause incidences. In this study, we regarded the critical day length as the day length in which a half of the individuals in the population entered diapause.

\section{Night interruption}

To determine the phase position of the $\varphi_{\mathrm{i}}$, larvae reared under short-day conditions were exposed to a night interruption photoperiod. Mature larvae (5 days 
after larviposition), reared under a short-day photoperiod of LD 11.5:12.5 $\mathrm{h}$ from their embryonic stage, were placed in wet chips at a depth of $10 \mathrm{~mm}$. This treatment, called water treatment, prolongs the larval photosensitive period and enables us to expose the larvae to photoperiods for a determined time (Goto and Numata, 2009ab). In this study, the scotophase was systematically interrupted by a $2 \mathrm{~h}$ light pulse under LD 10:14 h conditions, i.e. LDLD 10:x:2:(12-x) h, for 4 days. In our preliminary experiments, we had maintained flies under LD 10:14 $\mathrm{h}$ and exposed them to LDLD 10:x:2:(12-x) $\mathrm{h}$ but it did not work, as a light pulse did not avert diapause, possibly because the photoperiod was very short for some strains. Therefore, we maintained flies under LD 11.5:12.5 $\mathrm{h}$ and exposed them to LDLD 10: $x: 2:(12-x) \mathrm{h}$ for night interruption. After water treatment, larvae were transferred onto dry wood chips at a 30-50 $\mathrm{mm}$ depth under LD 11.5:12.5 h, to allow them to pupariate and pupate.

The most effective phases for diapause aversion were assessed from the lightpulse response curve. When two phases were similarly effective (less than 5\% difference), both are regarded as the most effective phase.

\section{Judgment of the developmental status and sex}

Twenty or more days after larviposition, the anterior tip of the puparium was removed using fine forceps, to assess the diapause status (diapause or nondiapause) 
according to the standard described by Fraenkel and Hsiao (1968). After the assessment, the pupae were pulled out from the puparia to determine the sex. Their sexes were judged based on the differences in the size of the fourth and fifth tarsomeres on the middle legs, the fifth tarsomere is greatly larger than the fourth in males but not in females (Tagaya et al., 2010).

\section{RESULTS}

\section{Latitudinal and sexual differences in critical day lengths}

The photoperiodic response curves of four geographic strains are shown in Fig. 3B. Flies exhibited a clear photoperiodic response, where short days elicited a high diapause incidence and long days elicited a low diapause incidence. Their critical day lengths were different, with the northern strains entering diapause under longer photoperiods, i.e. a clear latitudinal cline (Fig. 3B). Clear latitudinal clines were also observed when data of females and males were separately analysed (Fig. 3C-E). It is noteworthy that diapause incidences were significantly higher in males than females, at least under a certain photoperiod, in southern strains (OSK and NGS) ( $\chi^{2}$ test, $P<$ $0.05)$ but not in northern strains (OTR and UTM) $\left(\chi^{2}\right.$ test, $\left.P>0.05\right)$. Therefore, critical 
day lengths were longer in males than females in southern strains, whereas nearly identical in northern strains (Fig. 3E).

\section{Systematic scanning of the scotophase by a light pulse}

The systematic scanning of the scotophase by a $2 \mathrm{~h}$ light pulse was conducted on the NGS strain (Fig. 4). Previously, the same experiment has been done for the OSK strain, with similar but slightly different results obtained (Goto and Numata, 2009b). In both cases, the effects of a light pulse varied markedly among the phases of the scotophase. In the NGS strain, $1 \mathrm{~h}$ after scotophase onset, the diapause incidence of the population remained high, but the incidence dropped sharply when a light pulse was presented $2 \mathrm{~h}$ after scotophase onset. From 3 to $6 \mathrm{~h}$ after scotophase onset, the diapause incidence increased gradually. A light pulse presented 7-9 and $11 \mathrm{~h}$ after scotophase onset was ineffective in averting diapause. In contrast, the light pulse $10 \mathrm{~h}$ after scotophase onset was effective. The light-sensitive phase $2-5 \mathrm{~h}$ after scotophase onset is the circadian delay phase, in which the circadian clock delays its phase, and the lightsensitive phase $10 \mathrm{~h}$ after scotophase onset is the $\varphi_{\mathrm{i}}$. Diapause incidence in females was consistently lower than that in males, but a qualitative difference was undetectable in these light-sensitive phases, i.e. in both sexes the light-sensitive phase, in which the 
circadian clock delays its phase, occurred 2-6 h after scotophase onset and the $\varphi_{\mathrm{i}}$ occurred $10 \mathrm{~h}$ after scotophase onset.

To clarify the exact position of the $\varphi_{\mathrm{i}}$ in the late scotophase, a $2 \mathrm{~h}$ light pulse was systematically applied to the four geographic strains, 8.5-11 h after scotophase onset, with $15 \mathrm{~min}$ intervals. If the phase positions of the $\varphi_{i}$ were different among the geographical strains, the effective phases for diapause aversion by a light pulse are different among them. The most effective phases for diapause aversion among strains varied from 9.00 to $10.25 \mathrm{~h}$ after scotophase onset, i.e. the phases were 9.00-9.25, 9.50, 9.75-10.00, and 10.25 $\mathrm{h}$ after scotophase onset in the OTR, UTM, OSK, and NGS strains (Fig. 5, left columns), respectively. Therefore, the phase positions of the $\varphi_{\mathrm{i}}$ are qualitatively different among strains and a clear negative latitudinal cline of the phase positions of the $\varphi_{\mathrm{i}}$ was observed (Fig. 6). The negative latitudinal cline was also clear in the data of the females only and males only (Fig. 5, middle and right columns, respectively; Fig. 6). Although some small differences were detected, the most effective phases for diapause aversion were identical between the sexes in each strain. These results indicate that the phase positions of the $\varphi_{\mathrm{i}}$ are identical between the sexes and both sexes measure photoperiods in the same manner (Fig. 6). 


\section{DISCUSSION}

Danilevskii (1965) was the first to demonstrate the positive correlation between latitude and the critical photoperiod for diapause induction. He showed that the critical photoperiod increases by approximately $1 \mathrm{~h}$ with every $5-6^{\circ}$ increase in latitude in the knot grass moth, Acronicta rumicis. Since, such a latitudinal cline has been observed in several insect species (Bradshaw and Lounibos 1977; Kimura et al., 1993; Lankinen et al., 2013; Lehmann et al., 2015; Paolucci et al., 2013; Shimizu and Kawasaki, 2001; Wang et al., 2012). A latitudinal cline of the critical day length for diapause induction was also obvious in four geographic strains of S. similis in this study. The night interruption photoperiods reveal a clear latitudinal cline in the phase positions of the $\varphi_{\mathrm{i}}$. A strain from a higher latitude possesses a $\varphi_{\mathrm{i}}$ at an earlier stage in the scotophase than a strain from a lower latitude. These results indicate that the qualitatively different photoperiodic time measurement systems among geographic strains causally induce the latitudinal difference in the critical day lengths, i.e. Hypothesis I (Fig. 2B). Although a similar model has been proposed by Saunders (2013) and Meuti and Denlinger (2013), it has not been empirically tested.

The next question is how the $\varphi_{\mathrm{i}}$ is positioned at different phases in the scotophase among strains. At least two possibilities exist. One is based on the same phase angle of the $\varphi_{\mathrm{i}}$ in the circadian clocks showing different periods (Model A, Fig. 
2B1-3) and the other is based on qualitatively different phase angles of the $\varphi_{i}$ with no assumption of the circadian system variations (Model B, Fig. 2B4-6). In the model A, if the period of the clock of a group with a longer critical day length was shorter than that of another group with a shorter one, the $\varphi_{i}$ of the former is located at an earlier scotophase than that of the latter. Although we still do not fully understand the molecular mechanisms of the circadian clock involved in photoperiodic time measurement, the clock regulating photoperiodism appears to share many of the same genes as the circadian clock that regulates locomotor activity rhythms, i.e. the circadian clock genes including period (per), timeless (tim), cycle (cyc), Clock (Clk), and mammaliantype cryptochrome (cry-m or known as cry2) (Koštal, 2011; Saunders, 2011, 2016; Goto, 2013; Dolezel, 2015). It is well known that clock gene mutants show great variations in the circadian rhythmicity. For example, a null mutant of per shows a loss of circadian rhythmicity in adult eclosion and locomotor activity rhythms and several mutants in the per alleles shorten or lengthen the period of the clock (Konopka and Benzer, 1971). A recent study revealed that genomic regions containing circadian clock genes (per, cyc, and $c r y-m)$ are found to be associated with geographic variations in the photoperiodic diapause induction of a wasp (Paolucci et al., 2016). A close association between the geographic variations and genomic regions containing per and tim is also reported in a butterfly (Pruisscher et al., 2018). The conspicuous correlation between the clock gene 
haplotypes and photoperiodic induction are also reported (Mathias et al. 2007; Pegoraro et al, 2014; Tauber et al. 2007; Yamada and Yamamoto, 2011). Latitudinal clines of several properties of endogenous circadian rhythms, such as a negative latitudinal cline of circadian periods, are observed in Drosophila species (Lankinen, 1986, 1993). These results may support the model A (Hut et al., 2013). On the other hand, considerably low correlations between the period of the Nanda-Hamner rhythm (the period of the circadian clock involved in the photoperiodic response) with latitude and the critical day length were also reported in a mite and mosquito species (Vaz Nunes et al., 1990; Wegis et al., 1997; Bradshaw et al., 2006). In addition, no genetic association between the critical day length and circadian period was clearly demonstrated in the mosquito (Bradshaw et al., 2012). These results may support an idea that the phase angles of the $\varphi_{i}$ qualitatively differ among the geographic strains with no assumption of the circadian clock variations, i.e., the Model B (Fig. 2B4-6). We are unable to determine which models are applicable to the photoperiodic response in S. smilis. It is crucial to investigate the properties of the circadian system involved in the photoperiodic induction among these geographic strains, such as the NandaHamner rhythm and the phase response curve of the circadian clock involved in photoperiodism (Bradshaw et al., 2012; Saunders, 1976). 
It is noteworthy that the sexual differences in the critical day length for the photoperiodic induction of diapause was smaller in the northern S. similis strains than the southern ones. Although a similar trend is also reported in the cotton bollworm Helicoverpa armigera (Shimizu and Fujisaki, 2002), how the sexual difference in the photoperiodic response changes along with latitude has received little attention in other insect species. A small sexual difference at high latitudes might be derived from the overwintering risks, as proposed by Denlinger (1981). At higher latitudes, autumn is shorter and winter comes earlier. Thus, reproduction during autumn, by avoiding diapause, would be riskier and the need to enter diapause to survive the winter would be high in both sexes. In this case, the sexual difference in the trade-off between high fertility and environmental security becomes negligible, which promotes the disappearance of the sexual difference in the critical day length. It is of interest to compare the latitudinal sexual difference of the photoperiodic response curves with the latitudinal difference of the risks associated with overwintering.

In southern strains (OSK and NGS), sexual differences in both diapause initiation and incidence were obvious such that males had a longer critical length, as reported by Tagaya et al. (2010). The present study reveals that the phase positions of the $\varphi_{\mathrm{i}}$ are identical between sexes, irrespective of the difference in the critical day lengths. These results support Hypothesis II, i.e. a sexual difference in the quantitative 
evaluation of photoperiods and accumulation of the substance (Fig. 2C; Tagaya et al., 2010). This quantitative manner could alter the critical day length with no requirement of changing the central circadian clockwork (Meuti and Denlinger, 2013). Development is arrested in diapausing pupae, and these individuals do not progress to the next metamorphic stage (adult) until diapause is terminated and the prothoracic gland (PG) releases ecdysteroids (Denlinger et al., 2012). Prothoracicotropic hormone (PTTH), from neurosecretory cells (PTTH cells) in the brain, is responsible for the activation of the PG (Smith and Rybczynski, 2012). In a moth in pupal diapause, the PTTH cells are less excitable and neurophysiologically silent, i.e. high voltage thresholds, low input resistance, and few spontaneous action potentials (Tomioka et al., 1995). Richard and Saunders (1987) investigated the PTTH activity of the brain, which is assessed by in vitro activation of the PG cells to produce and release ecdysteroids, in diapausing flesh fly, Sarcophaga argyrostoma. They revealed that brain extracts from diapausing pupae have as much, or more, PTTH activity than brain extracts from non-diapausing pupae. Similarly, PTTH activity is higher in larvae of a flesh fly Boettcherisca peregrina destined for pupal diapause than in those destined for direct development (Moribayashi et al., 1992). These results indicate that the PTTH is present in the brains of individuals destined for diapause and even during diapause, but it is simply not secreted. Mizoguchi et al. (2013) clarified in the cabbage army moth, that a low PTTH titre in 
diapause pupae was promoted by the inhibition of PTTH secretion, not by PTTH production. Together with these results, we predict that the PTTH cells of S. similis males will easily be silenced and the release of PTTH from neurosecretory cells will be more easily inhibited, compared with females, even under the same short photoperiod. It is of great interest to investigate the sexual difference in the neurophysiological states of the PTTH cells, such as voltage threshold, input resistance and the number of spontaneous action potentials, under the same photoperiodic conditions.

The latitudinal difference in the critical day lengths involves qualitative variations in the photoperiodic time measurement system itself, whereas the sexual difference is possibly based on the quantitative photoperiodic time measurement and counter systems (Fig. 7). Thus, S. similis uses distinct mechanisms to induce the variations in the critical day lengths for seasonal events. However, we still do not know why flies adopt such distinct mechanisms for intraspecific variations in the critical day length for the photoperiodic induction of diapause. The circadian system is crucial for adapting to daily environmental cycles, and thus, governs a range of behaviours and physiological systems, such as locomotor activity, eclosion, sleep, phototaxis, learning, memory, feeding, metabolism, chemosensation, courtship, mating, and immunity (Allada and Chung, 2010). Changing properties of the circadian system would cause an alteration of these rhythms. The mechanism inducing the sexual difference found in 
the flesh fly can alter the critical day length for the photoperiodic induction of diapause, without changing the circadian properties of many output processes, and thus this might be an easier or less costly option for adjusting the critical day lengths (Meuti and Denlinger, 2013). A latitudinal cline of the circadian clock gene alleles has been reported in various insect species (Paolucci et al., 2016; Pruisscher et al., 2018; Tauber et al., 2007; see also Hut et al., 2013), suggesting insects need to adjust their circadian properties along with latitude. The mechanism inducing the latitudinal cline of the critical day length might simply be dependent on the latitudinal cline of the properties of the circadian system.

\section{ACKNOWLEDGMENTS}

This study is supported in part by JSPS KAKENHI (Grant-in-Aid for Scientific Research) Grant Number 16K08101 and the Osaka City University (OCU) Strategic Research Grant 2016 and 2017 for Basic Researches to SGG. We thank Editage (www.editage.jp) for English language editing of the manuscript submitted for publication. 


\section{CONFLICT OF INTEREST STATEMENT}

The authors declare that there is no conflict of interest.

\section{REFERENCES}

Allada R and Chung BY (2010) Circadian organization of behavior and physiology in Drosophila. Annu Rev Physiol 72: 605-624.

Bean DW, Dalin P and Dudley TL (2012) Evolution of critical day length for diapause induction enables range expansion of Dirhabda carinulata, a biological control agent tamarisk (Tamarix spp.). Evol Appl 5: 511-523.

Butler GD, Wilson LT and Henneberry TJ (1985) Heliothis virescens (Lepidoptera: Noctuidae): Initiation of summer diapause. J Econ Entomol 78: 320-324.

Bradshaw WE, Armbruster PA and Holzapfel CM (1998) Fitness consequences of hibernal diapause in the pitcher-plant mosquito, Wyeomyia smithii. Ecology 79:1458-1462.

Bradshaw WE, Emerson KJ and Holzapfel CM (2012) Genetic correlations and the evolution of photoperiodic time measurement within a local population of the pitcher-plant mosquito, Wyeomyia smithii. Heredity 108:473-479. 
Bradshaw WE, Holzapfel CM and Mathias D (2006) Circadian rhythmicity and photoperiodism in the pitcher-plant mosquito: can the seasonal timer evolve independently of the circadian clock? Am Nat 167:601-605.

Bradshaw WE and Lounibos LP (1977) Evolution of dormancy and its photoperiodic control in pitcher-plant mosquitoes. Evolution 31:546-567.

Danilevskii AS (1965) Photoperiodism and Seasonal Development of Insects, Oliver and Boyd, Edinburgh.

Danks HV (1987) Insect Dormancy: An Ecological Perspective, Biological Survey of Canada, Ottawa.

Denlinger DL (1971) Embryonic determination of pupal diapause in the flesh fly, Sarcophaga crassipalpis Macquart. J Insect Physiol 7:1815-1822.

Denlinger DL (1972) Induction and termination of pupal diapause in Sarcophaga (Diptera: Sarcophagidae). Biol Bull 142:11-24.

Denlinger DL (1981) Basis for a skewed sex ratio in diapause destined flesh flies. Evolution 35:1247-1248.

Denlinger DL, Yocum GD and Rinehart JP (2012) Hormonal control of diapause. In Insect Endocrinology, LI Gilbert, ed, pp 430-463, Elsevier BV Academic Press, Waltham, Massachusetts. 
Dolezel D (2015) Photoperiodic time measurement in insects. Curr Opin Insect Sci 7:98103.

Ellers J and van Alphen JJM (2002) A trade-off between diapause duration and fitness in female parasitoids. Ecol Entomol 27:279-284.

Fraenkel G and Hsiao C (1968) Manifestations of a pupal diapause in two species of flies, Sarcophaga argyrostoma and S. bullata. J Insect Physiol 14:689-705.

Gibbs D (1975) Reversal of pupal diapause in Sarcophaga argyrostoma by temperature shifts after puparium formation. J Insect Physiol 21:1179-1186.

Gnagey AL and Denlinger DL (1984) Photoperiodic induction of pupal diapause in the flesh fly, Sarcophaga crassipalpis: embryonic sensitivity. J Comp Physiol B 154:9196.

Gomi T, Nagasaka M, Fukuda T and Hagihara H (2007) Shifting of the life cycle and life-history traits of the fall webworm in relation to climate change. Entomol Exp Appl 125:179-184.

Goto SG (2013) Roles of circadian clock genes in insect photoperiodism. Entomol Sci $16: 1-16$

Goto SG and Numata H (2009a) Alteration of the pupal diapause program and regulation of larval duration by photoperiod in the flesh fly Sarcophaga similis Meade (Diptera: Sarcophagidae). Appl Entomol Zool 44:603-609. 
Goto SG and Numata H (2009b) Possible involvement of distinct photoreceptors in the photoperiodic induction of diapause in the flesh fly Sarcophaga similis. J Insect Physiol 55:401-407.

Goto SG and Numata H (2014) Insect Photoperiodism. In Insect Molecular Biology and Ecology, KH Hoffmann, ed, pp 217-244, CRC Press, Boca Raton.

Hut RA and Beersma DGM (2011) Evolution of time-keeping mechanisms: early emergence and adaptation to photoperiod. Phil Trans R Soc B 366:2141-2154.

Hut RA, Paolucci S, Dor R, Kyriacou CP, Daan S (2013) Latitudinal clines: an evolutionary view on biological rhythms. Proc R Soc B 280:20130433.

Kimura MT, Bessho A, and Dai Z-H (1993) The influence of gene flow on latitudinal clines of photoperiodic adult diapause in the Drosophila auraria species-complex. Biol J Linnean Soc 48:335-341.

Konopka RJ and Benzer S (1971) Clock mutants of Drosophila melanogaster. Proc Natl Acad Sci USA 68:2112-2116.

Koštál V (2011) Insect photoperiodic calendar and circadian clock: Independence, cooperation, or unity? J Insect Physiol 57:538-556.

Kurahashi H and Ohtaki T (1979) Induction of pupal diapause and photoperiodic sensitivity during early development of Sarcophaga peregrina larvae. Jpn J Med Sci Biol 32: 77-82. 
Lankinen P (1986) Geographical variation in circadian eclosion rhythm and photoperiodic adult diapause in Drosophila littoralis. J Comp Physiol A 159:123142.

Lankinen P, Tyukmaeva VI and Hoikkala A (2013) Northern Drosophila montana flies show variation both within and between cline populations in the critical day length evoking reproductive diapause. J Insect Physiol 59:745-751.

Lehmann P, Lyytinen A, Piiroinen S and Lindström L (2015) Latitudinal differences in diapause related photoperiodic responses of European Colorado potato beetles (Leptinotarsa decemlineata). Evol Ecol 29:269-282.

Lewis RD and Saunders DS (1987) A damped circadian oscillator model of an insect photoperiodic clock. I. Description of the model based on a feedback control system. J Theor Biol 128: 47-59.

Mathias D, Jacky L, Bradshaw WE and Holzapfel CM (2007) Quantitative trait loci associated with photoperiodic response and stage of diapause in the pitcherplant mosquito, Wyeomyia smithii. Genetics 176:391-402.

Matsuo Y (2006) Cost of prolonged diapause and its relationship to body size in a seed predator. Funct Ecol 20:300-306.

Meuti ME and Denlinger DL (2013) Evolutionary links between circadian clocks and photoperiodic diapause in insects. Integ Comp Biol 53:131-143. 
Mizoguchi A, Ohsumi S, Kobayashi K, Okamoto N, Yamada N, Tateishi K, Fujimoto Y and Kataoka H (2013) Prothoracicotropic hormone acts as a neuroendocrine switch between pupal diapause and adult development. PLoS ONE, 8:e60824.

Moribayashi A, Kurahashi H and Ohtaki T 1992 Physiological differenciation of the ring glands in matue larvae of the flesh fly, Boettcherisca peregrine, programmed for diapause or non-diapause. J Insect Physiol 38:177-183.

Nelson RJ, Denlinger DL and Somers DE (2010) Photoperiodism: The Biological Calendar, Oxford University Press, Oxford.

Paolucci S, Salis L, Cermeulen CJ, Beukeboom LW and van de Zande L (2016) QTL analysis of the photoperiodic response and clinal distribution of period alleles in Nasonia vitripennis. Mol Ecol 25:4805-4817.

Paolucci S, van de Zande L and Beukeboom LW (2013) Adaptive latitudinal cline of photoperiodic diapause induction in the parasitoid Nasonia vitripennis in Europe. J Evol Biol 26:705-718.

Pegoraro M, Gesto J, Kyriacou CP and Tauber E 2014. Role for circadian clock genes in seasonal timing: testing the Bünning hypothesis. PLoS Genetics 10:e1004603.

Pittendrigh CS and Minis DH (1964) The entrainment of circadian oscillations by light and their role as photoperiodic clocks. Am Nat 98:261-294. 
Pruisscher P, Nylin S, Gotthard K and Wheat CW (2018) Genetic variation underlying local adaptation of diapause induction along a cline in a butterfly. Mol Ecol 27:3613-3626.

Richard DS and Saunders DS (1987) Prothoracic gland function in diapause and nondiapause Sarcophaga argyrostoma and Calliphora vicina. J Insect Physiol 33:385-392.

Sadakiyo S and Ishihara M (2012a) Cost of diapause on life-history traits under restricted resources in multivoltine bruchid Acanthoscelides pallidipennis (Coleoptera: Bruchidae). Ann Entomol Soc Am 105:422-426.

Sadakiyo S and Ishihara M (2012b) Cost of male diapause indirectly affects female reproductive performance. Entomol Exp Appl 143:42-46.

Saunders DS (1966) Larval diapause of maternal origin-II. The effect of photoperiod and temperature on Nasonia vitripennis. J Insect Physiol 12:569-581.

Saunders DS (1976) The circadian eclosion rhythm in Sarcophaga argyrostoma: some comparisons with the photoperiodic "clock". J Comp Physiol A 110:111-133.

Saunders DS (1978a) An experimental and theoretical analysis of photoperiodic induction in the flesh-fly, Sarcophaga argyrostoma. J Comp Physiol A 124:75-95.

Saunders DS (1978b) Internal and external coincidence and the apparent diversity of photoperiodic clocks in the insects. J Comp Physiol A 127:197-207. 
Saunders DS (1979) External coincidence and the photoinducible phase in the Sarcophaga photoperiodic clock. J Comp Physiol A 132:179-189.

Saunders DS (2002) Insect Clocks, 3rd edition, Elsevier Science, Amsterdam.

Saunders DS (2011) Unity and diversity in the insect photoperiodic mechanism. Entomol Sci 14:235-244.

Saunders DS (2013) Insect photoperiodism: Measuring the night. J Insect Physiol 59:110.

Saunders DS (2016) The temporal 'structure' and function of the insect photoperiodic clock: a tribute to Colin S. Pittendrigh. Physiol Entomol 41:1-18.

Saunders DS and Lewis RD (1987a) A damped circadian oscillator model of an insect photoperiodic clock. II. Simulations of the shapes of the photoperiodic response curves. J Theor Biol 128:61-71.

Saunders DS and Lewis RD (1987b) A damped circadian oscillator model of an insect photoperiodic clock. III. Circaidian and “hourglass" responses. J Theor Biol 128:73-85.

Shimizu K and Fujisaki K (2002) Sexual differences in diapause induction of the cotton bollworm, Helicoverpa armigera (Hb.) (Lepidoptera: Noctuidae). Appl Entomol Zool 37:527-533. 
Shimizu T and Kawasaki K (2001) Geographic variability in diapause response of Japanese Orius species. Entomol Exp Appl 98:303-316.

Smith W and Rybczynski R (2012) Prothoracicotropic Hormone. In Insect Endocrinology, LI Gilbert, ed, pp 1-62, Elsevier BV Academic Press, Waltham, Massachusetts.

Tagaya J, Numata H and Goto S (2010) Sexual difference in the photoperiodic induction of pupal diapause in the flesh fly Sarcophaga similis. Entomol Sci 13:311319.

Tanaka K and Murata K (2016) Rapid evolution of photoperiodic response in a recently introduced insect Ophraella communa along geographic gradients. Entomol Sci 19:207-214.

Tanaka K, Murata K and Matsuura A (2015) Rapid evolution of an introduced insect Ophraella communa LeSage in new environments: temporal changes and geographical differences in photoperiodic response. Entomol Sci 18:104-112.

Tanaka M, Tachibana S-I and Numata H (2008) Sensitive stages for photoperiodic induction of pupal diapause in the flesh fly Sarocphaga similis (Meade) (Diptera: Sarcophagidae). Appl Entomol Zool 43:403-407.

Tauber MJ, Tauber CA and Masaki S (1986) Seasonal Adaptations of Insects, Oxford University Press, Oxford. 
Tauber E, Zordan M, Sandrelli F, Pegoraro M, Osterwalder N, Breda C, Daga A, Selmin A, Monger K, Benna C, Rosato E, Kyriacou CP and Costa R (2007) Natural selection favors a newly derived timeless allele in Drosophila melanogaster. Science 316:1895-1898.

Tomioka K, Agui N and Bollenbacher WE (1995) Electrical properties of the cerebral prothoracicotropic hormone cells in diapausing and non-diapausing pupae of the tobacco hornworm, Manduca sexta. Zool Sci 12:165-173.

Urbanski J, Mogi M, O’Donnell D, DeCotiis M, Toma T and Armbruster P (2012) Rapid adaptive evolution of photoperiodic response during invasion and range expansion across a climatic gradient. Am Nat 179:490-500.

Vaz Nunes M, Koveos DS and Veerman A (1990) Geographical variation in photoperiodic induction of diapause in the spider mite (Tetranychus urticae): a causal relation between critical nightlength and circadian period? J Biol Rhythms 5:47-57.

Vinogradova EB (1976) Embryonic photoperiodic sensitivity in two species of fleshflies, Parasarcophaga similis and Boettcherisca septentrionalis. J Insect Physiol 22:819-822. Wang X-P, Yang Q-S, Dalin P, Zhou X-M, Luo Z-W and Lei C-L (2012) Geographic variation in photoperiodic diapause induction and diapause intensity in Sericinus montelus (Lepidoptera: Papilionidae). Insect Sci 19:295-302. 
Wiklund C, Wickman P-O, Nylin S (1992) A sex difference in the propensity to enter direct/diapause development: A result of selection for protandry. Evolution 46:519-528.

Yamada H and Yamamoto M-T (2011) Association between circadian clock genes and diapause incidence in Derosophila triauraria. PLoS ONE 6:e27493. 


\section{FIGURE LEGENDS}

Figure 1. Photoperiodic response curve (A) and conceptual diagrams of the external coincidence model (B and C) and counter system (D), which describes the photoperiodic response of the flesh fly. (A) The flesh fly enters pupal diapause in response to a short-day photoperiod. The critical day length is defined as the length of the light fraction, of the light-dark cycle, that separates a strong long-day response from a strong short-day response in the photoperiodic response curve. (B) Photoperiodic time measurement in the external coincidence model is based on an oscillation of the circadian clock, which sets its phase at dawn and dusk, and on the position of the photoinducible phase $\left(\varphi_{\mathrm{i}}\right)$ in the latter half of the scotophase. During long summer days, the $\varphi_{\mathrm{i}}$ is exposed to light and a long-day response is induced (B1). During short autumnal days, the $\varphi_{\mathrm{i}}$ falls in the dark phase, eliciting a short-day response (B2). When a light pulse is applied during the early scotophase, the clock delays its phase and thus the $\varphi_{\mathrm{i}}$ is pushed out to the main photophase to be exposed to light. This gives rise to a long-day effect (B3). A light pulse during the late scotophase directly illuminates the $\varphi_{\mathrm{i}}$, to induce a long-day response (B4). When the scotophase was systematically scanned with a light pulse, 2 light-sensitive phases averting diapause were observed; the former is the circadian delay phase promoting the phase 
delay of the clock, and the latter is the $\varphi_{i}(B 5)$. (C) Photoperiods are also quantitatively evaluated. In this model, insects quantitatively produce a hypothetical diapauseinducing substance based on the photoperiods, according to how long the $\varphi_{i}$ is exposed to light (C1-C5). (D) The substance is accumulated in the counter system. When the level of the accumulated substance exceeds a certain 'internal threshold' diapause induction is determined, whereas nondiapause development is determined when the accumulation is lower than the threshold

Figure 2. Two groups with different critical day lengths (A) and two hypotheses that explain the difference (B and C). (B) Hypothesis I assumes the presence of a qualitative difference in the photoperiodic time measurement between groups; i.e. a difference in the phase positions of the $\varphi_{i}$. Under this hypothesis, the $\varphi_{i}$ of a group with a longer critical day length (group $\mathrm{x}$ ) is positioned at an earlier phase of the scotophase, than that of a group with a shorter critical day length (group y). This can be achieved by the same phase angle of the $\varphi_{\mathrm{i}}$ in the circadian clocks with different periods (Model A; B13) or by different phase angles of the $\varphi_{\mathrm{i}}$ in the circadian clocks with the same periods (Model B; B4-6). In both of these, the group with a longer critical day length (group x) enters diapause even under a moderately long day length (B1 and B4). During moderately short days, the group with a shorter critical day length (group y) also 
enters diapause because its $\varphi_{\mathrm{i}}$ is in the dark phase (B2 and B5). The systematic interruption of the scotophase by a light pulse will detect this qualitative difference in the phase positions of the $\varphi_{\mathrm{i}}(\mathrm{B} 3, \mathrm{~B} 6$, and B7). (C) Hypothesis II assumes, not qualitative, but quantitative differences in the photoperiodic time measurement and counter systems. Under this assumption, the positions of the $\varphi_{\mathrm{i}}$ are identical between groups (C1-C4), but they respond to the photoperiod differently. A group with a longer critical day length (group $\mathrm{x}$ ) synthesises larger amounts of the hypothetical diapause-inducing substance in response to photoperiods than another group with a shorter critical day length (group y) (C5). The substance is accumulated daily in the counter system. The substance easily exceeds the internal threshold in the group with a longer critical day length, in contrast to the group with the shorter critical day length (C6), and thus the former and latter likely enters and avoids diapause, respectively, even under the same photoperiod. The main concept is whether the accumulated amount of the substance exceeds the threshold or not, and thus, this hypothesis includes the same synthetic rates of the substance with different internal thresholds between the groups

Figure 3. Locations where the strains of Sarcophaga similis originated (A), their photoperiodic responses (B-D), and the relationship between the latitude of where the 
strain originated and critical day length (E). Photoperiodic response curves obtained from the data of both sexes $(B ; n=36-182)$, females only $(C ; n=17-86)$, and males only (D; $n=19-96)$ of the OTR (circles), UTM (triangles), OSK (squares), and NGS (diamonds) strains at $20^{\circ} \mathrm{C}$ are shown. Flies were reared under the photoperiod continuously. Diapause incidences under LD 12:12, 13:11, and 14:10 h conditions were separately compared with Tukey-type multiple comparisons for proportions. The same letters indicate statistical insignificance $(P>0.05)$

Figure 4. Effect of the systematic interruption of the scotophase by a light pulse on the diapause incidence of the NGS strain of Sarcophaga similis. Diapause-destined larvae were placed on wet wood chips (water treatment) and exposed to night interruption photoperiods. After water treatment, larvae were transferred onto dry wood chips to allow them to pupariate and pupate $(n=23-77)$. Asterisks indicate significant differences between the sexes $\left(\chi^{2}\right.$ test, $\left.P<0.05\right)$

Figure 5. Effects of interrupting the late scotophase with a light pulse on the diapause incidence of the OTR (the top row), UTM (the second), OSK (the third), and NGS (the bottom) strains of Sarcophaga similis. Data from both sexes (left column), those from females only (middle column), and those from males only (right column) are shown ( $n$ 
= 32-137). The same letters indicate statistical insignificance (Tukey-type multiple comparisons for proportions, $P>0.05)$. The phase that was most effected by the light pulse during the late scotophase is also indicated

Figure 6. The relationship between the latitudes of where the strain originated and the phase positions of the $\varphi_{\mathrm{i}}$ after scotophase onset. The data shown in Figure 6 were arranged to show the latitudinal cline. The average was plotted when two phases were similarly effective (less than $5 \%$ difference). The upper panel is derived from the data of both sexes and the lower panel is derived from the data of females (grey marks and grey solid line) and males (open marks and black broken line). The OTR (circles), UTM (triangles), OSK (squares), and NGS (diamonds) strains are shown

Figure 7. Modules inducing latitudinal and sexual differences in photoperiodic induction of diapause in Sarcophaga similis. The latitudinal difference in the critical day lengths involves qualitative variations in the photoperiodic time measurement system itself, whereas the sexual difference is based on the quantitative photoperiodic time measurement and counter systems 
A: Photoperiodic response curve

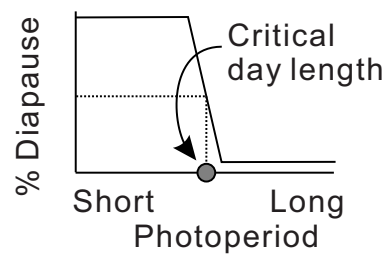

D: Counter

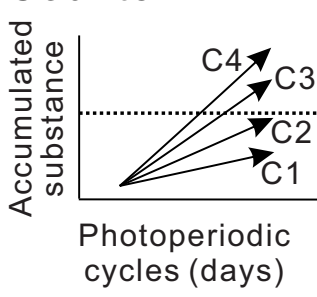

Short-day

response

$\uparrow$ threshold Long-day response

\section{B: External coincidence model}

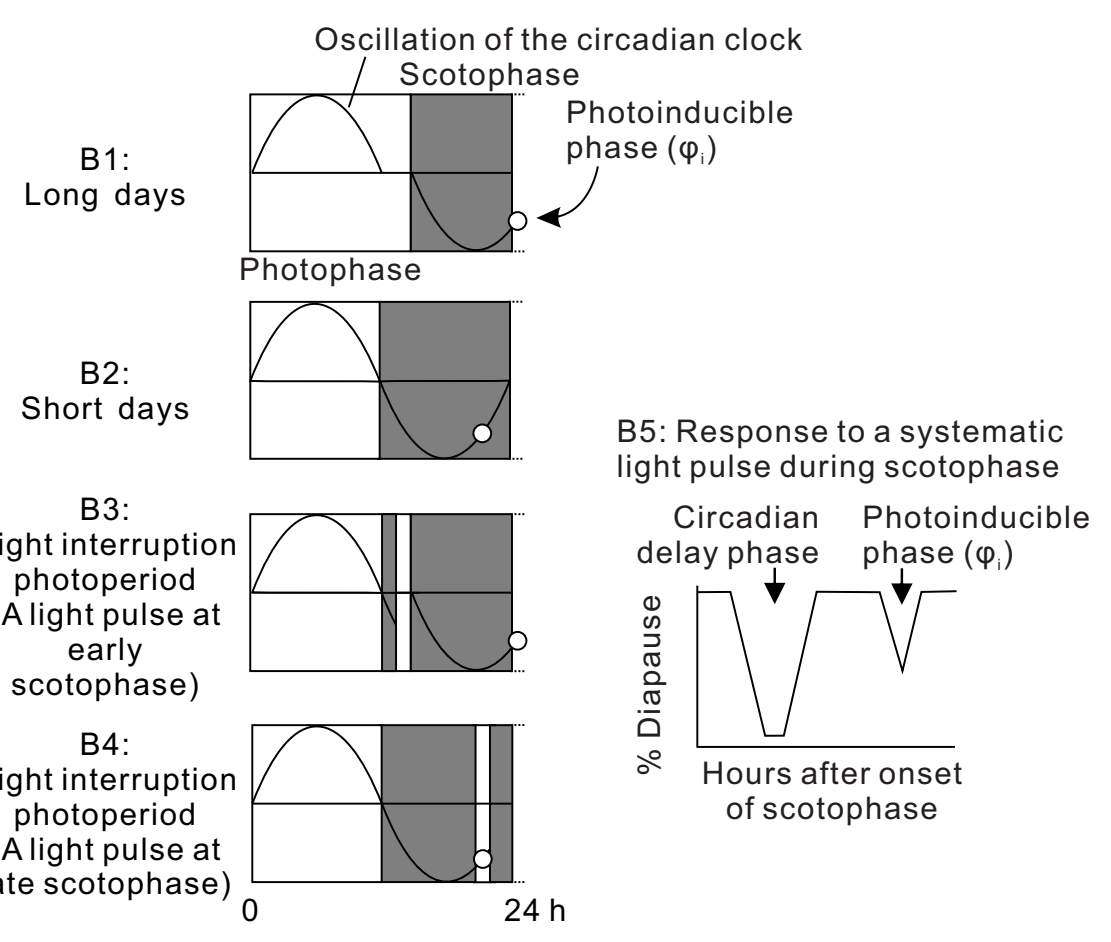

\section{C: Quantitative evaluation of photoperiods by the external coincidence model}

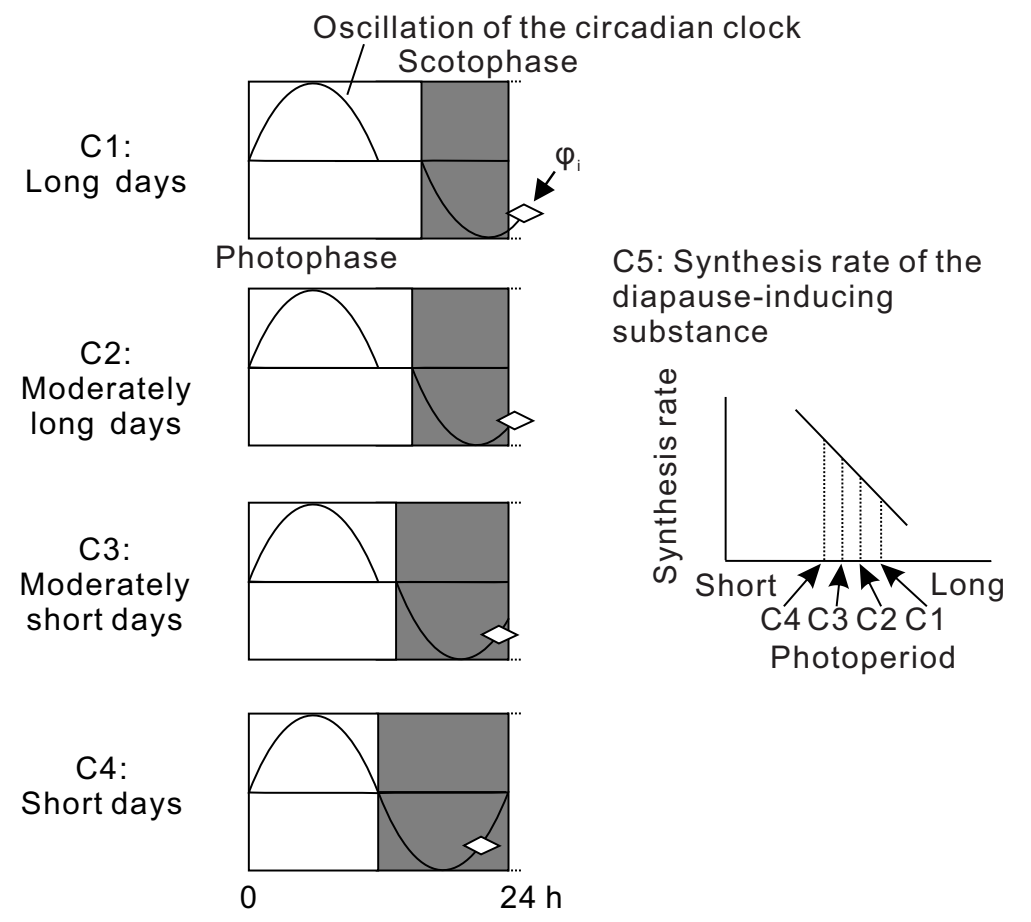


A: Difference in the critical day length

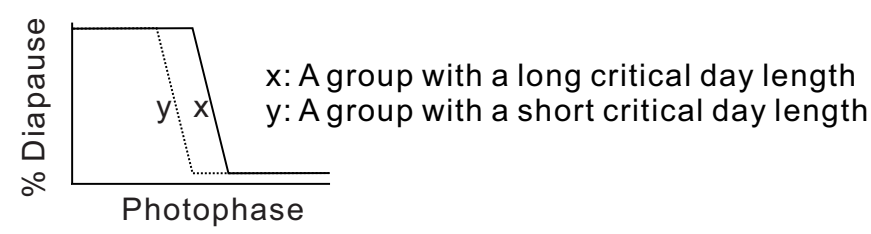

B: Hypothesis I: Qualitative difference in the photoperiodic time measurement

Oscillation of the circadian clock
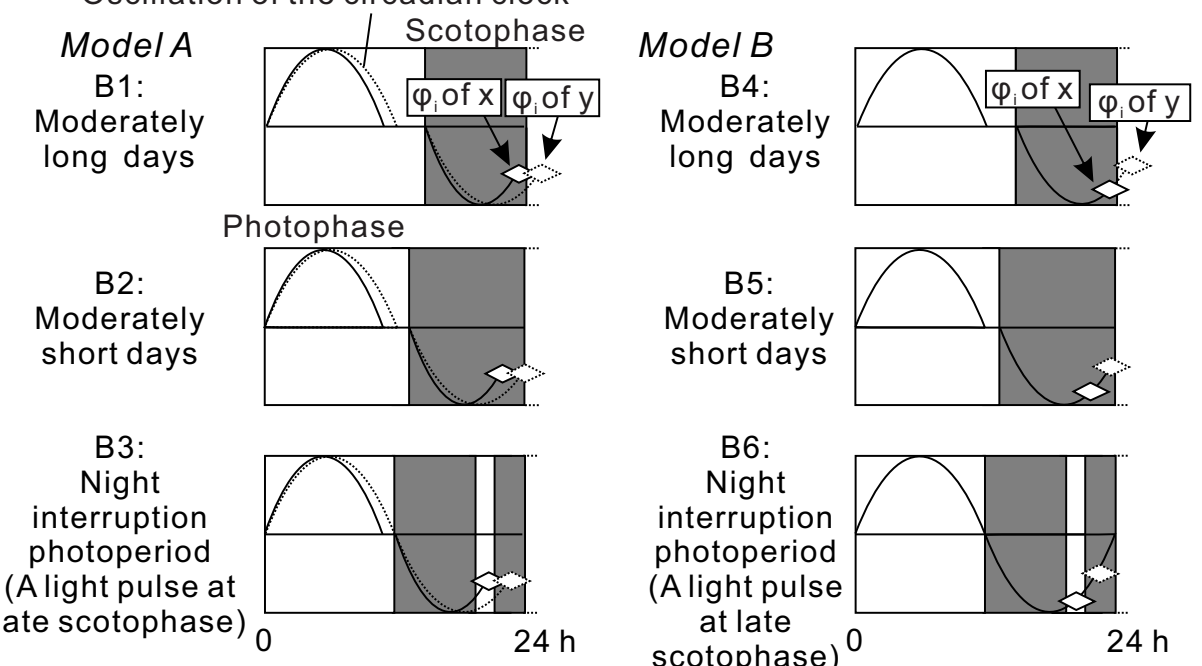

B6:

Night interruption photoperiod (A light pulse at late scotophase) 0

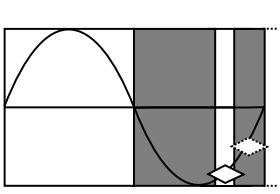

$24 \mathrm{~h}$
B7:

Responses to a light pulse

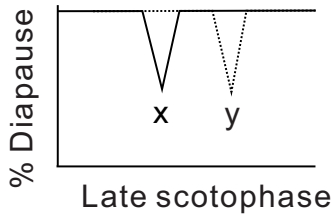

\section{C: Hypothesis II: Quantitative difference in the photoperiodic time measurement \& counter}

Photoperiodic time

measurement Oscillation of the circadian clock

C1:

Long days

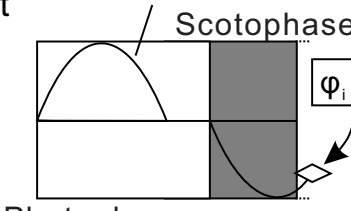
$\varphi_{\mathrm{i}}$ of $\mathrm{x} \& \mathrm{y}$

C5: Synthesis rate of the diapause-

\section{Photophase}

C2:

Moderately long days

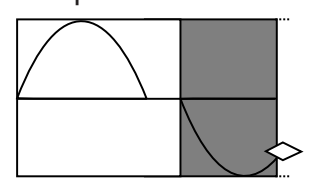

C3:

Moderately short days

C4:

Short days
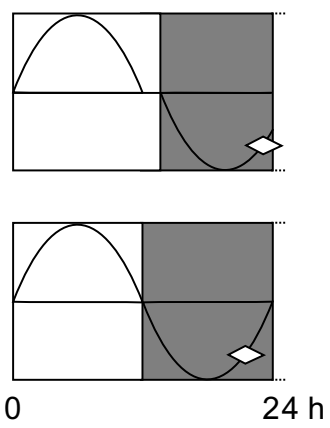

inducing substance

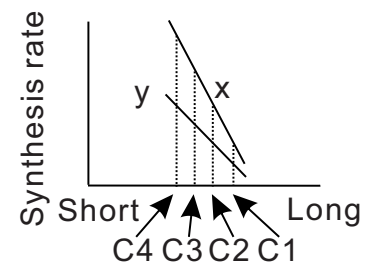

Photoperiod

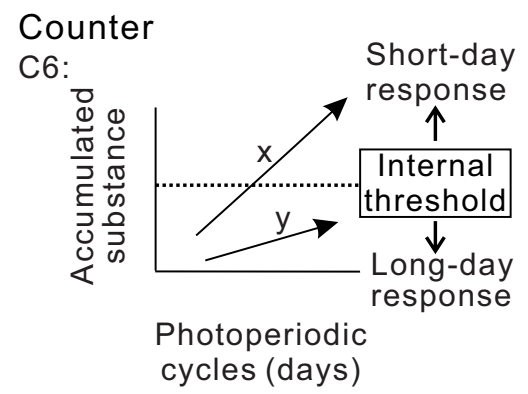



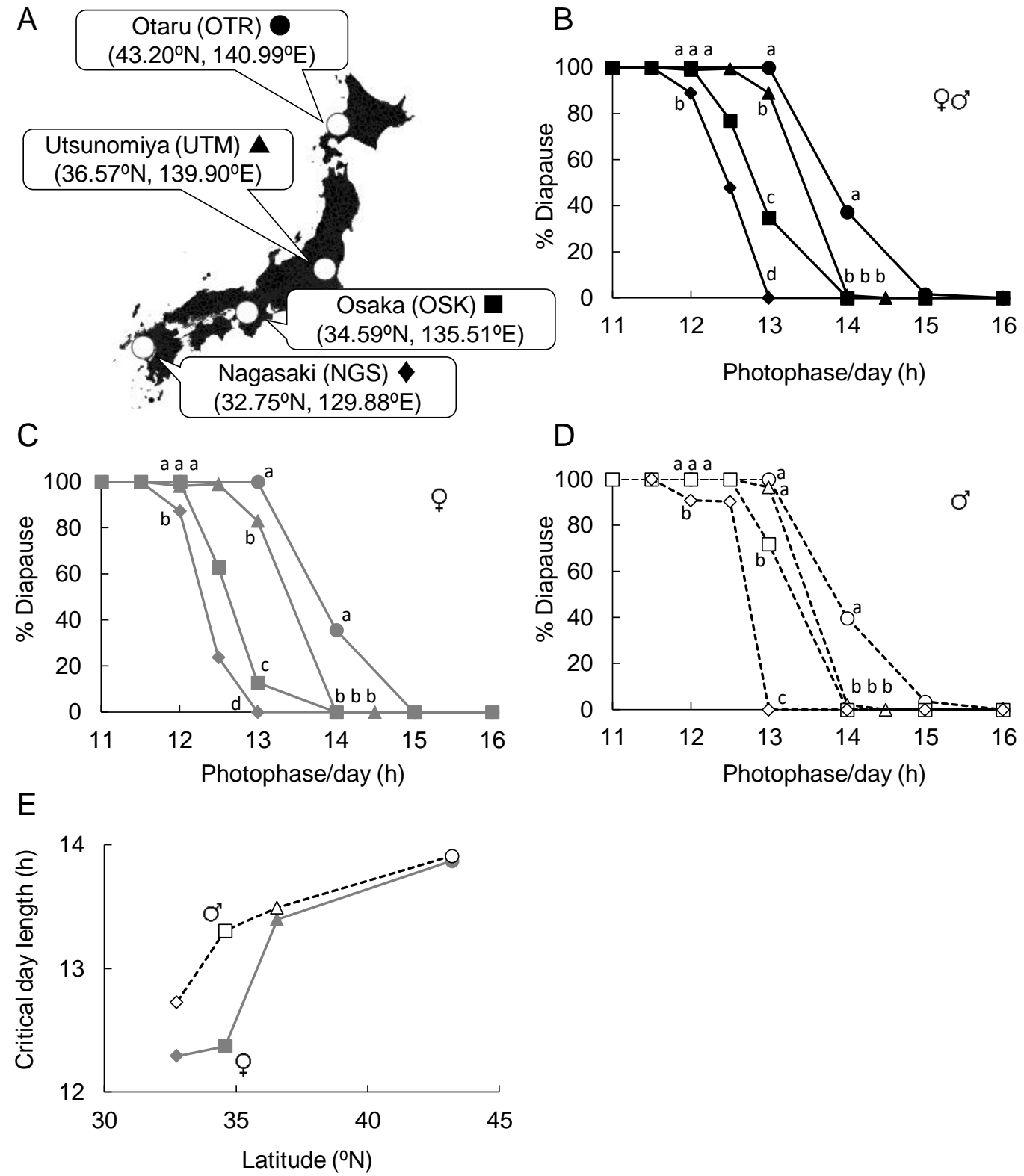

Fig. 3 


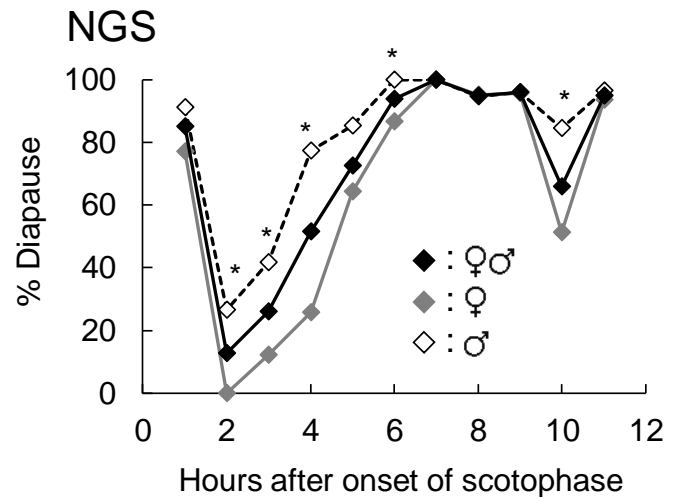

Fig. 4 

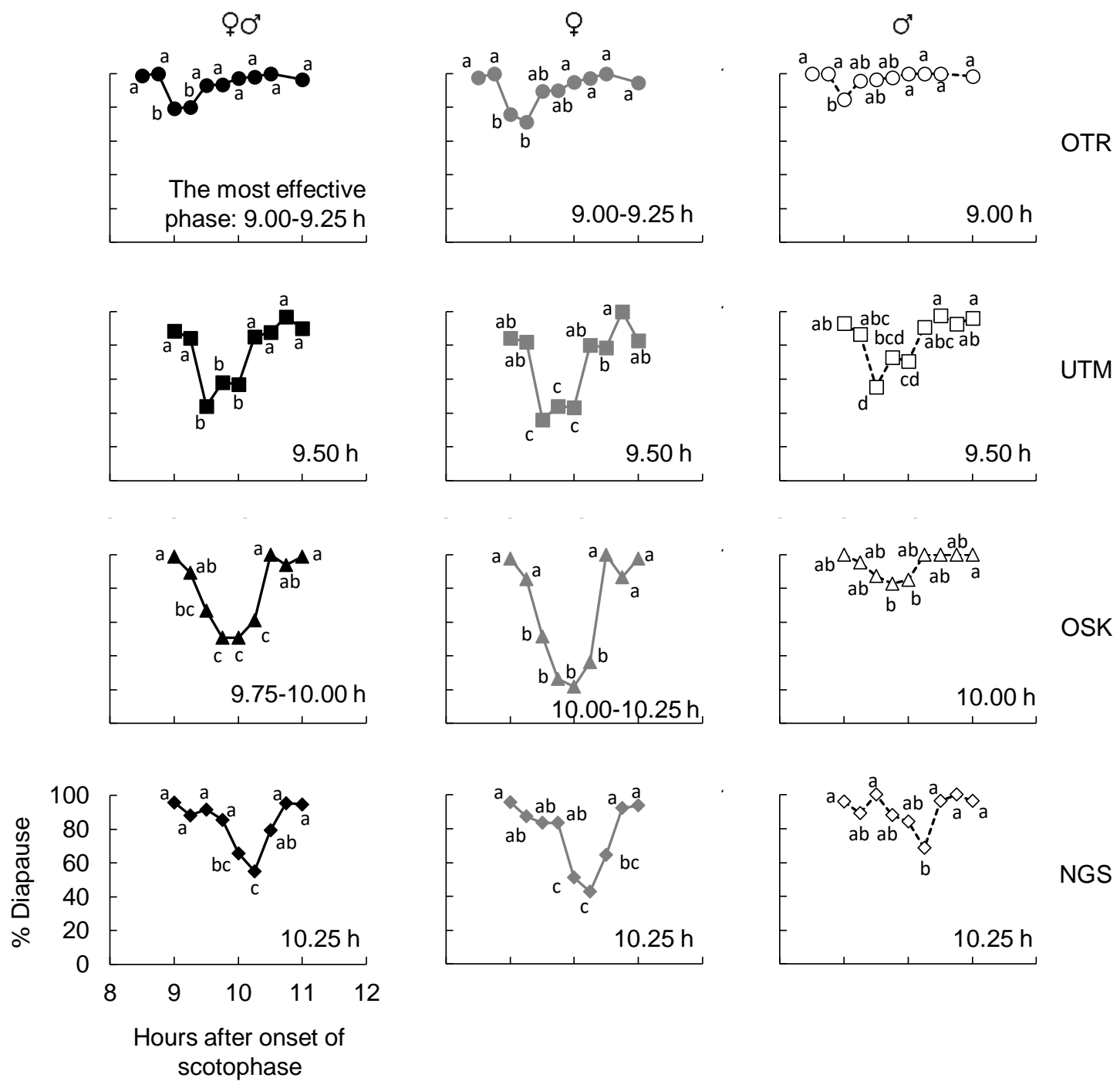

ours after onset of cotophase 

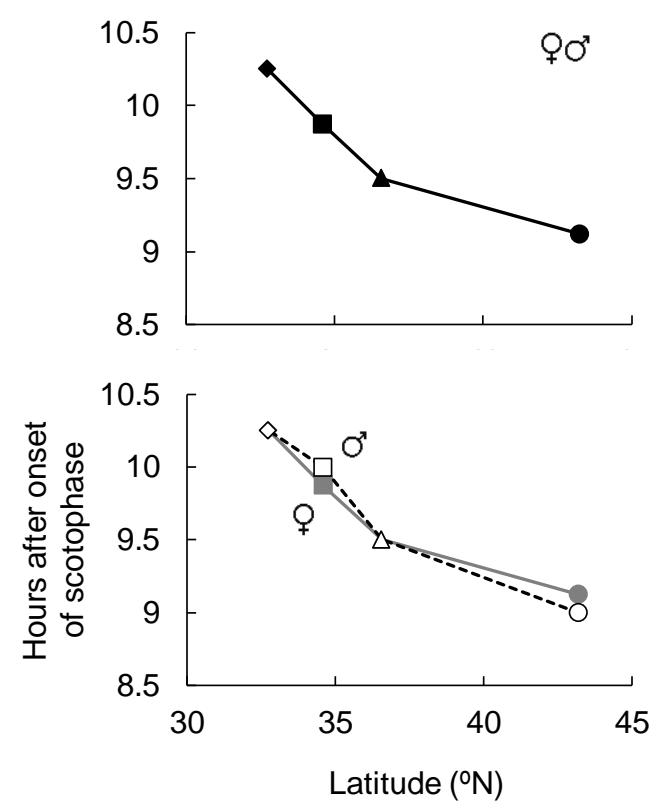

Fig. 6 


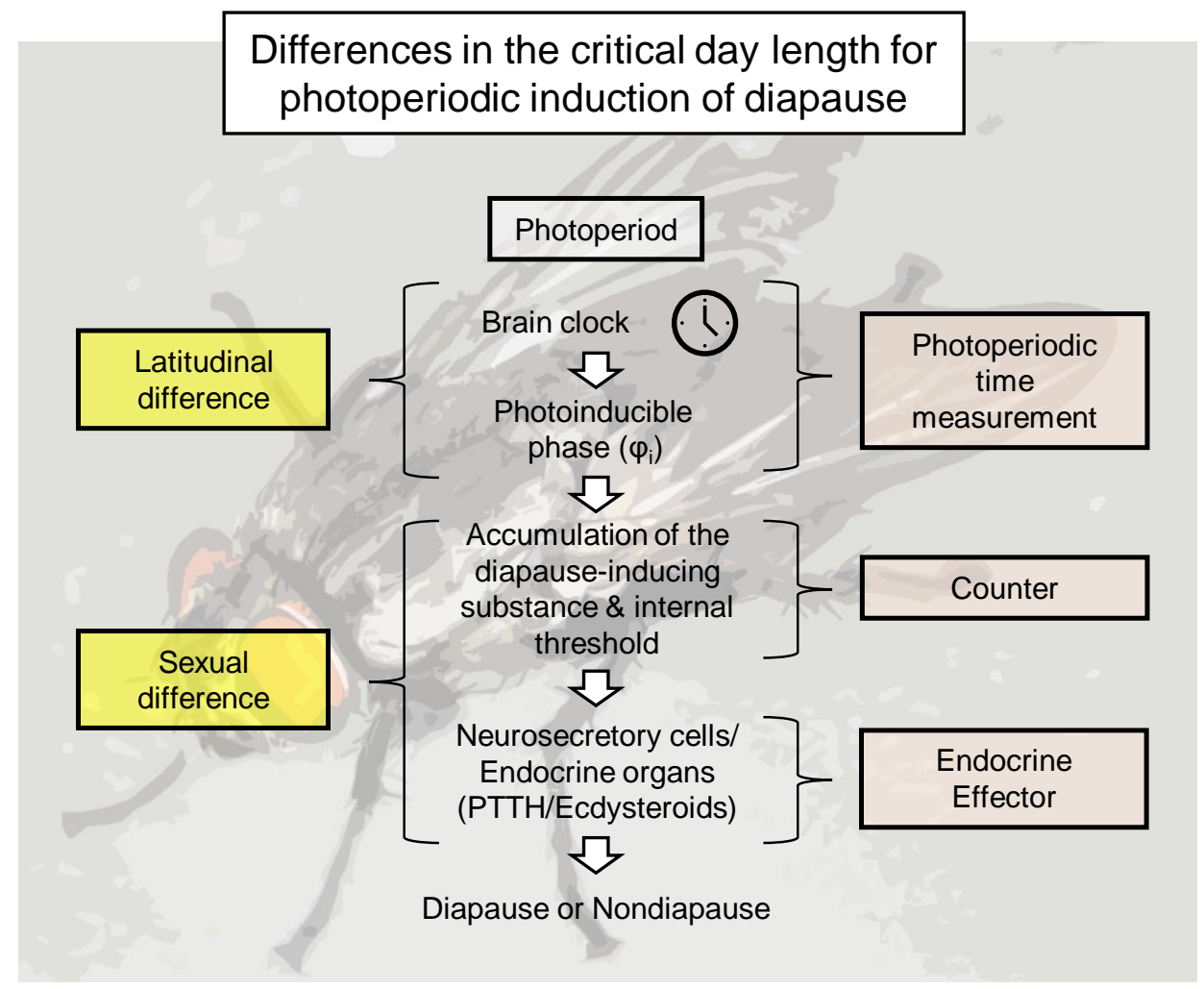

